\title{
Exploring Food-Related Beliefs and its Impact on Preschool-Aged Children in Pokhara in Nepal: A Qualitative Review
}

\author{
Jib Acharya ${ }^{1 *}$, Edwin van Teijlingen ${ }^{1}$, Jane Murphy $^{1}$, and Basma Ellahi ${ }^{2}$ \\ ${ }^{1}$ Faculty of Health and Social Sciences, Bournemouth University, England \\ ${ }^{2}$ Faculty of Health and Social Care, University of Chester, Riverside Campus, Chester, England
}

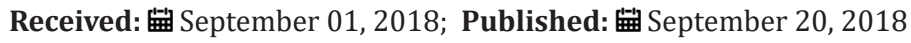

*Corresponding author: Jib Acharya, Faculty of Health and Social Sciences, Bournemouth University, 19-21 Bournemouth House, Christchurch Road. Lansdowne Bournemouth, Dorset, England, BH1 3LT
Abstract
a) poverty,
b) knowledge,
c) policy,
d) beliefs about breastfeeding,
e) food beliefs, and
f) beliefs and cultural influences.

Background: The main purpose of this study was to explore the experience of food beliefs among mothers from Kaski district of the Federal Democratic Republic of Nepal. In Nepal, there are few studies conducted based in the social sciences.

Aim: This study explores food beliefs among poor mothers related to feeding their offspring and the wider community's view.

Materials and Methods: Participants were recruited from relevant community associations via a purposive sampling technique. Fifty participants took part in seven focus group discussions to explore their food beliefs and the effect of how and what they feed their children as well as related health-seeking behaviors. The thematic analysis identified six key themes:

Results: Many participants thought that illiterate and underserved populations such as those which exist in Nepal are generally exposed due to financial scarcity, poor knowledge and strongly rooted cultural practices, and beliefs. This study found 'diversified views' as a major barrier (Figure 1) to food and health-seeking behaviours. Some groups recognised the negative effects of existing beliefs and taboos. However, the spiritual healers highlighted the importance of linking beliefs with cultural and religious norms and values. They showed the complex relationships between food and health-seeking behaviours and food recommendations with financial status and the perceived cultural practices of society.

Conclusion: The results illustrate the need for policy-makers and health professionals to take into consideration the environmental, social and cultural contexts of the mothers who are targeted for nutrition and food recommendations.

Keywords: Malnutrition; Beliefs; Taboos; Religion; Culture; Ethnicity; Poverty; South Asia

\section{Introduction}

Across the different geographical regions of Nepal, there is a diversity of food habits and cultural and traditional food beliefs which affect health and nutrition in both positive and negative ways. [1-3]. Several misconceptions and beliefs regarding food and healthseeking behaviours still exist in the community [4-7]. Sufficient healthy food is important for children and pregnant and lactating women, but insufficient food consumption may lead to children becoming malnourished. Pregnant women are more likely to have a low birth weight baby, develop anaemia, have complications during delivery and there is a high mortality risk for both mothers 
and children. It is challenging to change food and health-seeking behaviours and it presents a substantive burden for the community as well as the nation [7-10]. Mothers in Nepal misunderstand the role of healthy eating in combating nutritional problems that can lead to the inappropriate feeding of young children which in turn can lead to several complications, particularly in preschool-aged children $[7,9,10]$. For example, it has been reported that long-term breastfeeding may cause breast cancer, mothers may become ugly, and breasts may reduce in size and thus mothers are discouraged to breast feed infants long-term. Conversely, Jernström and colleagues [11] highlighted that breastfeeding reduced post-partum bleeding and increase the rate of recovery from labour among new mothers [12]. Also, for young children, breastfeeding prevents urinary tract infections and gastrointestinal, chest, ear and allergies, reduce the incidence of infant mortality, and decreases the risk of overweight and obesity among infants, children and adolescents $[12,13]$. These are examples of the contradictions which Nepalese mothers face. As a consequence, thereare several food beliefs and misconceptions in Nepal and which result in practices by people in the wider Nepalese community which impacts on food and health-seeking behaviours and may account for the high prevalence of malnutrition (as undernutrition) [14].

Many studies and surveys conducted in Nepal have focused on the link between nutrition and maternal and child health $[1,4,15-19]$. Some explored the widespread belief that pregnancy is a natural condition that does not need any particular attention $[17,20]$. Christian et al. [3] reported women's eating habits during pregnancy in the rural Terai region of Nepal where an avoidance of eating green leafy vegetables, fruits and dairy products occurred and reduced portion sizes were consumed [3]. This study further cited the commonly held belief that if a woman eats more during pregnancy she will have a heavier baby which can cause problems during delivery $[1,4,16,21]$. Acharya and colleagues revealed that beliefs about healthy diet were strongly embedded with cultural and religious norms and values where everyone respects their culture and religion and that in particular food and health-seeking issues are interlinked with culture and religion [15].

Furthermore Biza-Zepro 10 highlighted that food consumption is influenced by socio-cultural factors within households, cultural attitudes towards various foods, methods of food preparation and child-rearing practices. In developing countries, several international organizations such as UNICEF, HKI (Helen Keller International), Save the Children, Feed the Future have contributed towards behavioural changes in relation to, diet and child feeding practices in Nepal, particularly in the remote parts to change traditional practice [22,23]. Children mostly eat in communal groups depending on sex and age; and this is more common in the southern belt of Nepal. Some authors suggest that Nepalese mothers are generally of the opinion that children should be fed as often as the child wishes to eat, $[24,25]$ whereas mothers suggest that it should be four times a day [26]. In Nepal, the employment ratio is also very poor and accounts for only $24 \%$ of the total population as nearly $76 \%$ of the population is engaged in agriculture $[27,28]$. Socio-cultural practices such as paying less consideration to the supplementary feeding of children, late weaning and poverty are major underlying issues of undernutrition among preschool-aged children [29,30]. Similarly, child health nutritional indicators are used to evaluate the quality of existing health services as well as the general health condition of the wider community [29] Thus, the culture of Nepal affects eating and health-seeking behaviours which in turn contributes to undernutrition [7,31-33].

There is a body of literature which supports this supposition including Pokharel et al. [34] who argues behavioural aspects, such as feeding behaviour, as being important for childhood nutrition [8]. What is interesting, however, is that most of the research does not critically examine why such insufficient behaviours are present in the communities studied. Onta [35] highlighted the lack of knowledge on well-being and health safety along with nutritious food and uptake of health services in Nepal [35]. This may be attributed partly to traditional/spiritual healers and their status in Nepalese society. During the child sickness, the majority of the poor community accesses the healer's services for their children and gets the diet-related advice from the healers. The diet-related suggestions which are given by the healers, these are based on their past experiences and knowledge. These suggestions are strongly associated with the religious and cultural norms and values. [3538]. These common misconceptions or misbeliefs are strongly embedded in the poor and underserved rural communities as well as in some urban areas because of migration from rural areas [38]. The spiritual and traditional knowledge supporters or advocates are usually Hindu, as is $87 \%$ of the Nepalese population. Most of Nepalese society uses traditional or spiritual healers as the first step of health care [19,38-42]. Many people especially priests, people with lower level of education, spiritual/traditional healers, and the elderly parents still believe in myths affecting their eating habits and health-seeking behaviour $[43,44]$.

There are many examples of superstitious beliefs and behaviours involving odd philosophies of causal relationships in South Asia $[45,46]$. For these food and health beliefs, essential qualities of objects are taken as magical and mystically and regarded as transferable from one object to another, for example through simple resemblance or through provisional contact [47]. A variety of such beliefs still persists in the twenty-first century [48-51]. Food habits and practices are strictly linked to the wider culture [52]. People follow codes of conduct in relation to food consumption, food preparation methods eating habits and food selection. Meals are taken daily and the timing and portion size of a meal eaten are pre-defined. Nepal is in many ways a traditional society affected by social hierarchy and patriarchy on many levels $[1,53,54]$. These systems are the major generator of diversified 
views (multiple opinions) particularly for food recommendations in children, pregnant and lactating women which in turn affect food and health-seeking behaviours [3]. This paper seeks to understand the food beliefs and health seeking behaviors and their impact for woman incorporating a community wide perspective which illustrates the context within which women in Pokhari in Nepal gain and utilise knowledge on these aspects. In particular the questions are: What are the maternal views about healthy food? What is the mothers' food and health-seeking behaviours towards preschool aged children? What are the major factors that affect food recommendations What are the common features of these views and the level of diversification? What is the impact of these on health-seeking behavior?

\section{Methods}

Qualitative research using focus groups and an interview guide was employed to address the research aim.59 Seven focus groups were conducted mostly at training or meeting rooms in local government offices and in local hotels. Three focus groups were literate (table 2) and all discussions were held in Nepali. We conducted one pilot study consisting of nine people in Kaski district which helps to review and refine the data collection tools. 60 The Nepal Health Research Council (NHRC) approved this study 60).

Seven focus groups were conducted with: policymakers, auxiliary nurse midwives, health workers, pharmacists, social workers, spiritual healers and members of a mothers' group. Two of the group's members (ANMs and Mothers group) were involved in maternal and infant health management, i.e. they provided care, support and conducted programme evaluations. The focus group explored a detailed perspective on cultural practices and food beliefs, barriers, major causes of undernutrition and personal experiences particularly linked to misconceptions and taboos. There were between four and eleven participants in each group. Since, four groups did not give permission to use audio recording and to be consistent the researcher did not record any of the groups. The discussions lasted between 90 to120 minutes, with all the data manually recorded by the record keepers. Four groups did not give the permission to use audio recording and to be consistent the researcher did not record any of the groups. These data were transcribed word-for-word, translated into English and analysed using thematic analysis [55,56]. Applying the elements of the conceptual framework of Marks et al. 2001 [57,58]. From the analysis process possible themes were identified and then sections of data were structured into themes and sub-themes [56,59]. The transcripts were analysed by the first author and verified by another researcher. Analysis process further refined and merged into six overarching themes. Quotes illustrating key themes were selected to support the findings.

\section{Results}

There were 50 participants, 33 men and 17 women in seven focus groups (Table 1). The pharmacists' group was the smallest whereas the health workers were the largest. The auxiliary nurse midwives, health workers, and policy people worked for the local government. The mother's group is involved in local government health services. Social workers were volunteers with the Nepal Red Cross Society. Pharmacists and spiritual healers were selfemployed. "Most focus group participants were male, Hindu and between the ages of 36 to 45 (Table 1). Similarly, 8\% participants were illiterate, $26 \%$ were literate and $66 \%$ were educated (Table 2). The thematic analysis resulted in six key themes:

Table 1: Demographics of FG participants including religious status.

\begin{tabular}{|c|c|c|c|c|c|c|c|c|}
\hline \multirow[b]{2}{*}{ Group Label } & \multicolumn{2}{|c|}{ Gender } & \multicolumn{4}{|c|}{ Age Group Ratio } & \multicolumn{2}{|c|}{ Religions } \\
\hline & Male & Female & $25-35$ & $36-45$ & $46-55$ & 56-65+ & Hindu & $\begin{array}{c}\text { Buddh/ } \\
\text { Christ. }\end{array}$ \\
\hline Pharmacists & 4 & 0 & 1 & 3 & 0 & 0 & 4 & 0 \\
\hline $\begin{array}{l}\text { Auxiliary } \\
\text { Nurse } \\
\text { Midwives } \\
\text { (ANMs) }\end{array}$ & 0 & 7 & 4 & 2 & 1 & 0 & 5 & 2 \\
\hline $\begin{array}{c}\text { Spiritual } \\
\text { Healers }\end{array}$ & 5 & 0 & 0 & 0 & 0 & 5 & 5 & 0 \\
\hline $\begin{array}{l}\text { Mothers' } \\
\text { Group }\end{array}$ & 0 & 7 & 2 & 3 & 1 & 1 & 3 & 4 \\
\hline $\begin{array}{c}\text { Health } \\
\text { Workers }\end{array}$ & 10 & 1 & 3 & 5 & 3 & 0 & 10 & 1 \\
\hline $\begin{array}{c}\text { Social } \\
\text { Workers }\end{array}$ & 9 & 0 & 3 & 2 & 3 & 1 & 9 & 0 \\
\hline Policy People & 5 & 2 & 3 & 3 & 1 & 0 & 7 & 0 \\
\hline Total $(n=50)$ & 33 & 17 & 16 & 18 & 9 & 7 & 43 & 7 \\
\hline
\end{tabular}


Table 2: Literacy status of FG participants.

\begin{tabular}{|c|c|}
\hline Literacy status & Percent (\%) (n=50) \\
\hline Illiterate & $8 \%$ \\
\hline Literate & $26 \%$ \\
\hline Educated & $66 \%$ \\
\hline Total & $100 \%$ \\
\hline
\end{tabular}
a) poverty,
b) knowledge,
c) policy,
d) beliefs about breastfeeding,
e) food beliefs, and
f) beliefs and cultural influences.

\section{Poverty}

All Focus Group Discussions (FGD) thought that poor, illiterate and underserved populations were vulnerable due to both poor education and knowledge about healthy food and had strongly embedded cultural beliefs.

Fruit, green vegetables and other healthy commodity were seen as expensive and those most disadvantaged were particularly affected by high-market prices. In the words of one mother, "I cannot buy healthy foods due to lack of money as well as high prices." (FGD: Mothers' Group). Whilst the policy-makers added: "There is no choice for healthy diets by low-income groups because they are unable to afford dairy product \& meat items thus children from these groups are more susceptible to undernutrition". (FGD: Policy People). In Nepal, women's status is generally low and even more so amongst the poorest. Poor women often work harder than men especially on the land and in the house. In some ethnic groups, they hardly have any time for child care and feeding. One of the health workers said: "I have no time to care of my daughter due to loads of house and office work. My daughter is being cared (for) by my mother-in-law whose mind is completely occupied by beliefs and taboos mainly on child care and feeding." (FGD: ANM). Whilst one policy-maker noted that it is more than just a lack of money but refers to a lack of fundamental issues: "Poor people of this area are deprived of basic capabilities rather than simply low income. They are also destitute of basic needs, rights and opportunities and suffering after the natural disaster." (FGD: Policy Maker).

\section{Knowledge}

This theme covers the level of mothers' knowledge about undernutrition as well as common healthy and non-healthy foods and availability in practice. This theme explores understanding the consumption of nutritious foods. It captures the cultural or religious implications behind societal beliefs and taboos. It centres around the question: "Why do beliefs and taboos frequently come into practice in very systematic behaviours and yet are also highly diversified into different platforms such as in ethnic groups, castes and religions?" Conflicting or diversified views were reported. For example, in one group colostrum feeding was encouraged and, in another group, it was rejected. Poor households did not focus on the condition of children, whereas rich households tried to attend to childhood problems urgently. This variation is due to different levels of knowledge. This theme is influenced by patterns of economic status among participants and focuses on the attitudes and practices making up the food environment.

In terms of knowledge or education about nutritious food, the FGDs revealed three different sub-themes. First, all groups suggested that the level of awareness about undernutrition was lacking in poor people. Lack of awareness is the main factor that contributed to poor eating habits that impacts on nutritional status such as non-exclusive breastfeeding, late and inappropriate childfeeding practices, inadequate diet and rest for women during pregnancy and insufficient health-seeking behaviours. Secondly, most participants also regarded the low level of knowledge as one of the major factors for undernutrition, for example, one of the ANMs said that: "I noticed one family who stayed next door to a sub-health post (SHP) in my working area, who had four children. The grandparents always used to recommend them ready-made foods. After one year these children - three out of four - were diagnosed with undernutrition by the SHP and sadly one died. After this episode, they realized that it was due to the regular feeding of ready-made food. People of this area have fairly poor knowledge on a healthy diet. It may be due to poverty and low level of education." (FGD: ANM).

Thirdly, knowledge about nutritious food is highly diversified in the district. A few mothers who live in an urban area, who are educated and rich, are highly knowledgeable about healthy food but still have poor knowledge on what is nutritious food, how to prepare it or preserve its nutritional value. One of the participants in the policy people group drew on his personal experience in observing that: "One of my neighbours who is well off and highly educated never buys green vegetables and fruits from the market because of food adulteration. He always grows them in his own garden. I have also experienced that many mothers from disadvantaged communities do not give priority to child ailments unless the condition of the child gets worse. This type of knowledge is widely practised in Nepal which is creating big problems in the society." (FGD: Policy makers).

All focus group participants, except the spiritual healers, independently voiced similar opinions about mothers' knowledge and perceptions on undernutrition.

\section{Policy}

The debate focused on existing health and food policies of Nepal. The groups of pharmacists and policy people were actively involved 
and shared their views on the discussion of policies. In contrast, the ANMs, mothers' group and spiritual healers did not discuss policy at all. The latter might be due to a lack of knowledge of health policy including nutrition because of their low socio-economic status and education compared to the other groups. Additionally, ANMs and mothers' group members may have been concerned that their voices might reach higher authorities and may result in losing their jobs. For the same reasons, lower level health workers were also observed to be silent or digress from the topic when discussing policy issues concerning higher health authorities. A major concern was about the free health care services being provided by the government. The pharmacists agreed that: "An officially free health care policy was mainly targeted at poor and marginalized groups in the society but in practice on the ground, high disparities remain, as rich people are getting more benefit than the poor. It is documented in government records that the service has been used by poor and disadvantaged groups but practically it was used by rich and politically influential people." (FGD: Pharmacist).

A policy for the provision of free health-care services at a government facility under the district hospital has been progressively introduced, reducing financial barriers to promote uptake by poor and marginalised groups. However, disparities remain as the less disadvantaged benefit more than the poor from such programmes.

The pharmacists also commented on the weaknesses of child and maternal health policy. The provision was considered to be very different between private and public health institutions and policy must be the same in both health arenas. The private health sector was not following government rules and regulations with regard to public health issues. There was a lack of supervision and monitoring. The pharmacists explained that the poorest women were really deprived of care at birth especially in rural areas. One member of this group stated that women: "are unfairly discriminated in the society and working institutions of this society. It has been seen that the mothers of malnourished children lack proper health care before and after birth because most of the time, mothers are expected to get back to family chores, such as working in the farm or the kitchen just after delivery and it affects the health of the mother and child and their nutrition." (FGD: Pharmacist).

\section{Beliefs About Breastfeeding}

Beliefs and attitudes concerning breastfeeding were also associated with wider cultural and traditional beliefs. Most of the female participants showed considerable interest in breastfeeding and felt that breast milk was better than any infant milk formula and an artificial food supplement. A typical comment was:

"I think breastfeeding is highly effective for children. My motherin-law and mother taught me the importance of breastfeeding and colostrum because there are a lot of rumours about breastfeeding around this society and people don't listen to the facts about breastfeeding and colostrum and they confronted themselves. I also have observed my mother's health which is associated with longterm breastfeeding in the past. She is still beautiful and healthy. (FGD: ANM).

Most women reported that they squeezed out colostrum due to negative cultural beliefs about colostrum as advised by their mothers-in-law, spiritual healers and some health workers.

"In my case, I could not feed colostrum to my children because my mother-in-law forced me to discard colostrum before starting breastfeeding. But I had repeatedly explained to her about the significance of colostrum for the new borne which I had learnt from FCHV (Female Community Health Volunteer) even she did not agree with me and I was compelled to discard it." (FGD: Mother Group).

\section{Food Beliefs}

The focus groups highlighted crucial issues about food beliefs. The mothers' group in the village is trusted by local women who share health issues. So, they are also respected by the poor and less educated community. A mothers' group member added that: "Many families, around my working area, believed that feeding pregnant women a lot of food will make delivery difficult, so they even reduce the amount of food once they notice the pregnancy. Thus, I have observed that many pregnant women are being prevented from (eating) healthy food." (FGD: Mothers' Group). The health workers, pharmacists and social workers expressed similar views. Overall participants shared their experiences about the status of healthy children. Obese or overweight children are believed to be healthy children and low weight babies signify the poor socioeconomic status of a family. One experienced social worker highlighted that: "In remote parts of Kaski white pumpkin, aubergine/eggplants, yoghurt, eggs, green leafy vegetables and some fruits are avoided in pregnancy due to fear of miscarriages. Some ethnic groups believe that these food items can cause harm to the unborn baby and pregnant women are also likely to get common colds and stomach problems which harm the baby. I also heard that in hospital some doctors are also advising women to avoid foods in some sort of conditions. I think these misconceptions generally came out from some of the Hindu scriptures and films as well." (FGD: Social Worker).

One mother from the mothers' group, who are using complementary vitamin packets distributed by the government, cited: "I have three children, for the first two, I avoided many healthy foods while pregnant because plenty of misconceptions existed about healthy food. During the third time I was pregnant, I did have a nutritious diet which caused a big problem during delivery due to having a larger baby. There are numerous negative beliefs which are associated mainly with children, women and sick people; even I could not remember them (all)." (FGD: Mothers' Group). 


\section{Beliefs and cultural influences}

With regard to health-seeking behaviours, most participants had similar views except for the spiritual healers. Most groups were able to propose possible reasons for the poor not accessing available health services discussing cost, time, the level of awareness of the household or right decisions and trust in the services. The healers explained the rationale for sacrificing animals as a pragmatic means to fulfil the patient's nutrient requirements. Some food beliefs are also found to be highly beneficial to undernourished children such as the reason behind advising scarifying animals. The mothers' group, ANMs and social workers discussed health-seeking behaviour from different angles and expressed their experiences more openly. But the group of spiritual healers were muted and expressed that:

"Existing health institutions are ultimately costly for poor people and worthless mainly in Bhut, Prait and Pichas (forms of the spiritual beliefs). Thus, people are knocking on our doors because all the mothers care about their children and want to escape from the problems as soon as possible. We are honest, kind and more familiar with the community and we have distinct knowledge on the matter." (FGD: Spiritual Healer). The mothers' group discussed the principles of household behaviour especially during the sickness of a child. They agreed that most mothers wait for the child to recover by itself and later they make a short visit to a healer and wait for some days. If the child does not recover it is only then that they consider attending existing health institutions. If they do not have enough money then the child stays at home. One of the members of the mothers' group stated that:

"I have heard a lot of voices from many mothers from the community while doing home visits at my clusters when, honestly speaking, the children's mothers expressed that they do not understand the message mainly on medication and diet which is being delivered by health workers." (FGD: Mothers' Group).

\section{Discussion}

This study was designed to evaluate the impact of maternal knowledge, attitudes and food beliefs about nutritious food among the preschool-aged children and their impact. This qualitative study found poverty, knowledge, existing food beliefs, policy issues and health-seeking behaviours are major factors which prevent mothers to recommend nutritious food their children. As per the National Plan of Action of Nepal 39, the key contributing factors to childhood nutrition were child care practices, women's status and household food security. Beliefs about healthy diet are strong in this area as well as throughout Nepal. Generally, the literature suggests people have a strong negative association with healthy diets however this study found there is no difference between educated and non- educated people on this subject. Educated people are also highly influenced by these social constructs and religious beliefs resulting in contradictions on what constitutes a healthy and nutritious diet and other related behaviors for maternal and child health.

Spiritual healers were assertive during the debate on beliefs and taboos whereas policy people group were relatively less interested in the issues as were pharmacists. All focus group participants presented negative and positive beliefs about food and healthseeking behaviours $[15,60]$. Food beliefs particularly, associated with children, whether positive or negative, are strongly embedded in Nepalese society and can be routinely found in most households [3]. It seems that mothers and senior women in the households maintain traditional food beliefs. In addition, religious people (e.g. healers/shamans, priests, monks, pastors), herbalists, quacks (fake doctors) and health workers also preserve traditional food beliefs in this community [53]. Unfortunate events or incidents that occur within a household or community as a result of consuming certain food items also helps to create or perpetuate negative beliefs about food [61]. For example, the majority of mothers in this society do not give pomegranate to children because they believe it causes constipation. This is an example of perception-based evidence which still exists in this community yet negatively influences healthy food given to children [62].

This study identified 'diversified views' as a major barrier 3 to food recommendations (Figure 1). These highlighted variables are commonly revealed to be the key/activating issues (Knowledge, Attitudes, Beliefs and Practices) about healthy foods which present positive, negative and neutral views that directly or indirectly control/influence food and health-seeking behaviours. Diversified views (DV) emerged when relatives, friends and neighbours visit the sick child at home. They generally collect information about causes of child sickness, shared similar past experiences and advise food and further health consultations. Diversified views emerge during visits to the sick child which ultimately confuses mothers resulting in them making inappropriate decisions, particularly for food and health-seeking behaviours [16]. This DV strongly emerges in childhood sickness. For example, if a child is sick at my (JA) home, my relatives, friends and neighbours visit the sick child and they collect information about the sickness of child or problem from the parents of the sick child. After collecting information about the problem, they share their past experiences relating to child sickness and advise me (JA) as the parent about food and healthcare support. 2 this may contradict existing knowledge and as a result, causes confusion for the parents.3,13 Thus, each visit of relatives, friends and neighbors results in a number of diversified views which causes delayed decision making in relation to food/ nutrition and health-seeking behaviors. 


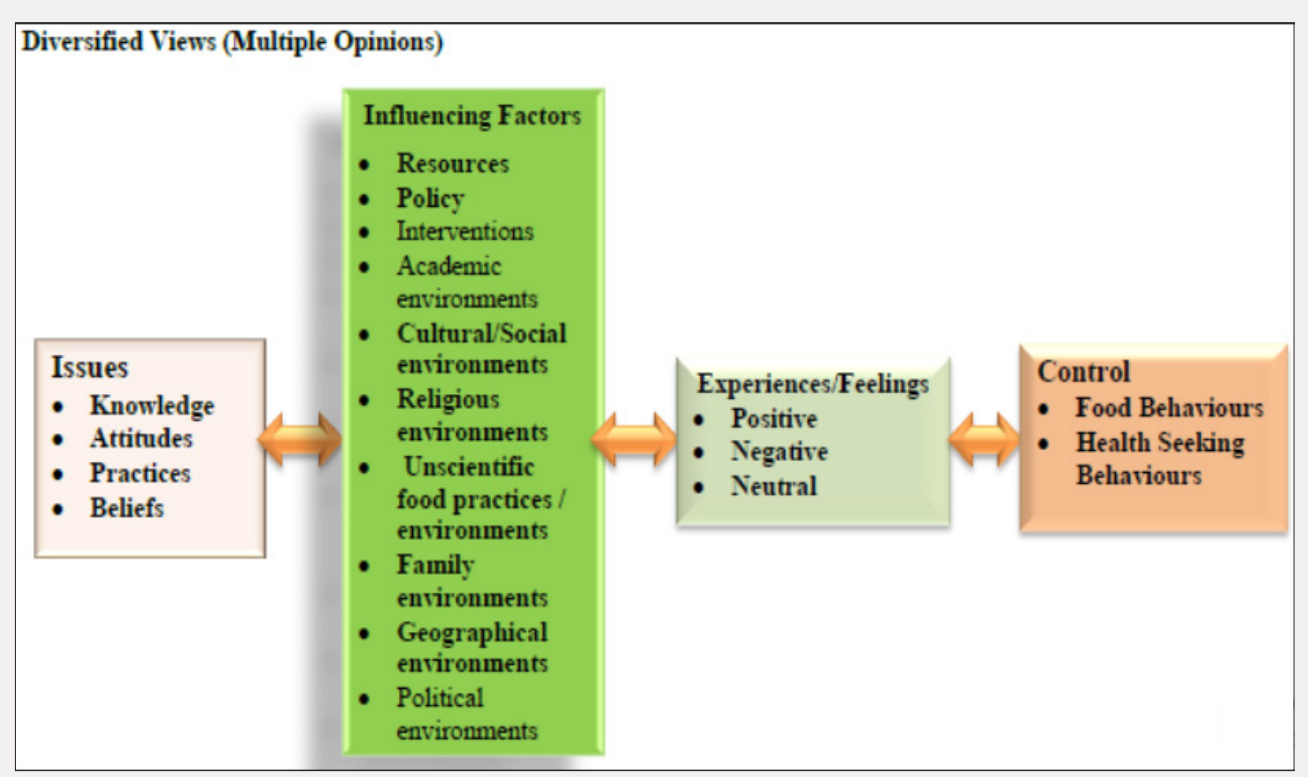

Note: The highlighted variables (on the diagram above (Influencing Factors' section) were strongly associated directly with the issues around knowledge, attitudes, practices and beliefs about the healthy diet. Non-highlighted variables are also important but to a less direct degree than the highlighted ones. Thus, the highlighted variables are commonly revealed to be the keyiactivating issues (Knowledge, Attitudes. Practices and Beliefs) about a healthy food which present positive, negative or neutral views that directly or indirectly control or influence the food and health-seeking behaviours of the mothers. The study found mostly the negative outcomes, towards the healthy diet. Diversified views (DV) emerged when the relatives, friends and neighbours visit the sick child home. They generally collect information about causes of child sickness during the visit. They also shared the similar past experiences with the parents of the sick child and advice for food and further health consultation based on their past experiences. It repeats in every visit of the visitors and which generates multiple opinions. And ultimately it creates a massive confusion among the mothers to take a proper decision to provide a healthy diet.

Figure 1: Diversified views (Multiple opinions).

Beliefs or misbeliefs about nutritious food have been continuously practised for centuries and these are strongly rooted in Nepalese society [39-41] In the past rural communities depended on spiritual healers (Jhankris) and herbal remedies (Jadi-buti). Nowadays, even after the development of a health care system in Nepal, most people still trust spiritual healers. Trusting in the spiritual faith is mainly due to beliefs, lack of awareness about healthy diet and poverty. In regard to beliefs, people still feel that they were made sick by devils such as witchcraft (Boksi) and ghosts (Bhut) and they go to healers regularly and use herbs extensively. Shamans or healers are also still practising numerous beliefs including those associated with foods in this community $[3,62,63]$. These healers have been continuously and staunchly supportive of this system as it is their main business, but as a consequence they perpetuate superstitions in this Nepalese society $[64,65]$. Thus, these beliefs are still deeply rooted in Nepalese custom and tradition as well as across other South Asian communities. This study also finds the similar notions regarding food beliefs which enrooted in the mothers' knowledge [3].

This study revealed several nutritional disorders still exist in this Nepalese community. For example, the mothers describe a number of terminologies for colostrum resulting in it being variously termed in the local language as bighaouti, pip, khoti, bikh, khil, and Phohar (yellow or thick substance, pus, poison and dirt). It is also highly debatable 35,43 Colostrum is also called pus in illiterate communities because of its resemblance to pus 57 . Many people still believe that the first milk, is an unsafe substance which should be discarded, yet scientifically it has evidenced benefits. This creates barriers to the feeding of first milk to the new-born $[15,17]$. Surprisingly, it has even been observed that even the owners of cattle do not give the colostrum to the calves of buffalo or cows in rural Nepal such is the depth of this belief. [41] The breastfeeding practice is contrary to the World Health Organization's recommendation that breastfeeding should start in the first hour after birth. Importantly, breastfeeding rates after expelling colostrum were high and expressing still stimulates a mother's milk supply. This may be why the practice of discarding colostrum, which is common in South Asian occurs, yet it is not a harmful as previously thought as it may stimulate feeding [42-71].

Generally, the Nepalese community is strongly influenced by Ayurveda and Unani medical practices. There are several food barriers such as food classifications including hot, cold and neutral foods, associated with these systems particularly while taking an Ayurveda and Unani medicines. On the other hand, these medication regimes are connected with cultural and religious norms and values which affect food and health-seeking behaviours. Examples of food 
beliefs, in Nepalese society, relates to that of food being considered pure or impure depending upon the food type, source, and handling $[60,41,61]$. Additionally, in Nepalese and even in Indian society or culture, the concept of cold and hot food is very significant and still exists, especially in rural communities $[41,60,66]$. Cold items are cooling foods such as fruits, green leafy vegetables and other types of vegetables $[41,60]$. Hot items such as meat, butter, soup of grains and fish are also prohibited when someone needs to cool off and they are preferred for extra energy and nutrition $[41,66,67]$. Hot and cold beliefs are held by many people in this community, $[60,40,61]$ together with people from South Asian and influence consumption patterns and behavour. [26,39] This concept is thought to originate from the Ancient Hindu medical system of Ayurveda and has been influential in medical practices since ancient times [60,64,68,]. The Unani medical system is also thought to have influenced popular thinking which is derived from the Ancient Greek humoral theory, was developed and adopted in areas that were under Muslim rule, for example, Gujarat, after assimilating medical knowledge from India and Persia [40,64]. As with Ayurveda, the Unani system includes hot and cold classification, however, the two classifications are not the same [40]. Generally, foods with a pungent, acidic or salty taste are considered 'hot, while those with a sweet, astringent or bitter taste, 'cold'. The basic concept of the Ayurveda and Unani medical systems is the maintenance of a dynamic balance in the body $[40,64]$.

In day-to-day life, little attention tends to be paid to the 'hot' and 'cold' classification of foods with their significance more so being felt during vulnerable physiological stages of life such as pregnancy, lactation or illness $[3,40]$. Adherence to beliefs also tends to be stronger in the rural community and among the nonliterate poor [45]. Modern-day Asian beliefs regarding hot and cold vary between countries and regions. Correct diet and digestion, together with a power of self-preservation are seen as influential to the maintenance of humoral balance [39]. The disease is thought to be caused by an imbalance in the humour. Many of the spices and seasonings used in South Asian food are believed to have therapeutic qualities, for example, turmeric is considered to have an antiseptic effect and garlic, ginger, cardamom, fenugreek, thyme, Asafoetida, and cloves are regarded as good for digestions and combating the bacterial effects often associated with food and the tropics $[3,64]$.

\section{Strengths and Limitations}

There are a number of strengths to this study. Very few researchers have addressed food and health-seeking behaviours amongst rural and urban mothers in Nepal. The qualitative methodology was one of the study's strengths allowing diverse groups such as policy people, health workers, spiritual healers, in their own words, to articulate their feelings and attitudes towards misconceptions and cultural practices about nutritious food and the topic could be explored in depth. The strengths of the method allowing the participants to discuss freely the issues under discussion created an honest sharing of views which enabled a deeper understanding of issues to be formed. Study contributes to the understanding of everyday beliefs about food and health, providing a basis for the idea that some phenomena in relation to food and health may be best understood as having paranormal features. Involving conceptual views, they go beyond mere associations and can coincide with scientifically valid views on the same topic $[39,40]$.

Despite these strengths due to the nature of qualitative research the generalizability of the research is limited to particular communities in Nepal. However, despite this the literature from elsewhere supports many of the findings from elsewhere and the conduct of the research ensures the rigour of the findings

\section{Conclusion}

Discussion subjects particularly relate to the community or household environments or situation and time mainly influencing the provision of a healthy diet to children. In regards to food beliefs, the older generation, particularly older women, and Hindu texts and films were generally blamed for influencing food behaviour and patterns of society. The group of spiritual healers was more favourable towards religious matters than the other groups. The healers explained the rationale for sacrificing animals as a pragmatic means to fulfil the patient's nutrient requirements. Generally, this community is strongly embedded with cultural and religious norms and values which support to practice the food beliefs routinely. Their responses highlighted the complexity of food socio-cultural beliefs and practices about the healthy diet. Most of the groups acknowledge social, cultural and environmental elements combined with the routine collection of data and continued support for these populations will be instrumental in maintaining and improving food and health-seeking behaviours in rural and urban mothers and the wider community. There were several misconceptions about the consumption of nutritious foods in the study population, which were generally derived from traditional and religious values and norms. These misconceptions were strongly associated with food and health behaviours and prevented mothers from recommending healthy foods. Therefore, most of the mothers' showed negative beliefs about healthy diets. Concerning food beliefs, multiple opinions (diversified views) exist, underpinning and reinforcing traditional and religious norms and values, so that generally mothers obtain instruction and opinions from neighbours, relatives and community members who think in stereotypical ways, causing wrong decisions due to diversified views and other influencing factors see (Figure 1). At the same time, the misconceptions and beliefs about healthy diets were also strongly linked with the multi-cultural, multi-religious, multi-ethnic and multi-lingual society in which every conceivable misconception 
is, therefore, possible, fuelling their diffusion, dissemination and adversely affecting people's food choices and health. Some food beliefs are also found to be highly beneficial to undernourished children such as the reason behind advising scarifying animals.

\section{Recommendation}

The recommendations resulting from this study may help resource poor groups in the community and local government to tackle the problems of food and health-seeking behaviours more effectively. The findings from this study reinforce the need to focus on nutritional issues in Nepal and conclude with further investigations particularly focusing on influential elements (Figure 1) including ethnic issues. The study strongly recommends shortterm, medium-term and long-term strategies targeting to the community level, government agency, and donors' agency including academicians. and in particular addressing the importance of clear evidence-based practices; addressing this knowledge gap has become a priority. Thus, implementing extensive mass awareness programme focusing on cultural and religious issues which are strongly associated with healthy diets. Implementation of the $3 \mathrm{P}$ awareness strategy is recommended for this purpose which focuses on the policy level, professional level and community level as an extensive mass awareness campaign.

\section{Ethical Approval/Consideration}

The NHRC and the Bournemouth University Research Ethical Committee (BUREC) both approved this study (ref. no. 1438). BUREC has provided a recommendation letter to NHRC for final approval. Furthermore, written and verbal consent was obtained from the Pokhara Sub-Metropolitan City, Child Welfare Scheme Nepal and the District Public Health Office of Kaski. The enumerators withdrew any participants who did not complete or wished to withdraw from the study up until the point of analysis. Coding has been used to protect anonymity of participants.

\section{Acknowledgement}

The author would like to thank to the Sight and Life, Switzerland, Global Supplementary Grant Programme/Open Society Foundation-Europe and Bournemouth University for their generous support to carry out this study. The authors would like to convey their sincere thanks to Child Welfare Scheme Nepal, Pokhara Metropolitan City, Western Regional Health Directorate Pokhara, NEPAN Field Office Pokhara, Western Regional Health Training Centre Pokhara, Kaski District Public Health Office, GONESA Nepal, La' Grandi International College, Pokhara (especially the 3rd year BPH students) for their cooperation and support in data collection.

\section{Refrences}

1. Acharya O, Zotor F, Chaudhary P, Deepak K, Amuna P, Ellahi B (2016) Maternal Nutritional Status, Food Intake and Pregnancy Weight Gain in Nepal. Journal of Health Management 18(1): 1-12.
2. Adhikari R (2010) Food Utilization Practices, Beliefs and Taboos in Nepal.

3. Christian P, Bunjun Srihari S, Thorne-Lyman A, Khatry S, LeClerq S, et al. (2007) Eating Downin Pregnancy: Exploring Food-Related Beliefs and Practices of Pregnancy in Rural Nepal. Ecology of Food and Nutrition 45(4): 253-278.

4. Acharya B (2013) Food Belief and Practices During Postoperative Period Among Post Operative Patient. Academiaedu.

5. Bista K, Karki Y, Thapa K (2009) Myths and Misconceptions about Abortion Among Marginalized Underserved Community Journal of Nepal Medical Association.

6. Evans R (2015) 'Witchcraft' abuse cases on the rise.

7. Najar N, Sharma B (2015) 11 arrested following 'human sacrifice' in Nepal. The Hindu.

8. Horton R (2008) Maternal and child undernutrition: an urgent opportunity. 371(9608): 179.

9. Joshi S, Barakoti B, Lamsal S (2012) Colostrum Feeding: Knowledge, Attitude And Practice In Pregnant Women In A Teaching Hospital In Nepal.

10. Karkee R, Lee A, Khanal V, Binns C (2014) Infant feeding information, attitudes and practices: a longitudinal survey in central Nepal. International Breastfeeding Journal 9(1): 9-14.

11. Adhikari D (2013) Malnutrition is Alarmingly High in Nepal | NewSpotLight Nepal News Magazine. Spotlightnepalcom.

12. Jernstrom H, Lubinski J, Lynch H (2004) Breast-feeding and the Risk of Breast Cancer in BRCA1 and BRCA2 Mutation Carriers, Journal of National Cancer Institute 96(14): 1094-98.

13. Horta B, Bahl R, MartinésC J, Victora C (2007) WHO Evidence on the longterm effects of breastfeeding: Systematic reviews and meta-analyses.

14. (2016) MoHP, New Era, ICF. Nepal Demographic and Health Survey: Key Indicators. Kathmandu, Nepal: Ministry of Health, India, 2017.

15. Acharya J, Teijlingen E, Murphy J, Hind M (2017) Food belief practices amongst rural and urban mothers in Nepal. A qualitative overview Research gate.

16. Devkota M, Uprety A, Subedi N, Poudel R (2012) Identification of gaps and priority interventions for maternal nutrition in Nepal: A review child health division.

17. Odent P (2011) Early infant feeding and neonatal survival in Nepal: breastfeeding, colostrum and discarding of the first milk - UCL Discovery. Discoveryuclacuk

18. Pahari D, Bastola S, Paudel R (2011) Factors affecting retention of child health card in a rural area. Ncbinlmnihgov 9(2): 154-158.

19. Paudel R, Pradhan B, Wagle R, Pahari D, Onta S (2013) Risk Factors for Stunting Among Children: A Community Based Case Control Study in Nepal. Kathmandu University Medical Journal 10(3): 18-24.

20. Subedi M (2003) Healer choice in medically pluralistic cultural settings: An overview of Nepali medical pluralism. Occasional Papers in Sociology and Anthropology 8: 128-158.

21. Simkhada B, Porter M, Van Teijlingen E (2010) The role of mothers-inlaw in antenatal care decision-making in Nepal: a qualitative study. BMC Pregnancy and Childbirth 10(1): 34 .

22. (2014) HKI. Helping Families Grow Better Food: Improving Nutrition.

23. (2013) UNICEF. Capacity Building to Strengthen Local Government and Community Participation in Multi-Sectoral Nutrition Programmes in Nepal.

24. Sah N (2005) Determinants of child malnutrition in Nepal: A case analysis from Dhanusha, central Terai of Nepal. 
25. Subba S, Chandrashekhar T, Binu V, Joshi H, Rana M, et al. (2007) Infant feeding practices of mothers in an urban area in Nepal 5(1): 42-47.

26. (2006) GSCP. Supply Chain Sustainability Resources \& Practices - The UN Global Compact.

27. (2015) Global Finance Report. Global Finance Magazine - The Richest Countries in the World. Global Finance Magazine.

28. Hunter M, Shaw C (1989) Nutrition and the elderly. Nursing Standard 4(2): 38-40.

29. (2011) NPHC. National Population and Housing Census (National Report). National Planning Commission. 2012.

30. Nazni P, Pradheepa S, Hasan A (2010) Effects of weaning biscuits on the nutritional profile and the cognitive development in preschool children. Italian Journal of Pediatrics 36(1): 18.

31. Raheela F (1994) Disability in the Bengali community - Social Care Online. Scie-socialcareonlineorguk p. 12-13.

32. Bista K, Karki Y, Thapa K (2009) Myths and Misconceptions about Abortion Among Marginalized Underserved Community Journal of Nepal Medical Association.

33. Sreeramareddy C, Shankar R, Sreekumaran B, Subba S, Joshi H, et al. (2006) Care seeking behaviour for childhood illness- a questionnaire survey in western Nepal. BMC International Health and Human Rights 6(1): 7.

34. Raj Kumar Pokharel, Robin Houston, Philip Harvey, Ramu Bishwakarma, Jagannath Adhikari, et al. (2009) Nepal nutrition assessment and gap analysis final report.

35. Onta S (2003) The report of knowledge, practice and coverage final survey in Kanchanpur district Nepal, India.

36. Lobstein T, Davies S (2009) Defining and labelling 'healthy' and 'unhealthy' food.

37. Mishra V, Retherford R (2000) Women's education can improve child nutrition in India. Scholarspacemanoahawaiiedu. Natl Fam Health Surv Bull 15: 1-4.

38. Meyer-Rochow V (2009) Food taboos: their origins and purposes. Journal of Ethnobiology and Ethnomedicine 5(1): 18.

39. Stone L (1976) Concepts of illness and curing in a central Nepal village. Journal of the Institute of Nepal and Asian Studies 1976: 55-80.

40. Storer J (1977) 'Hot' and 'Cold' food beliefs in an Indian community and their significance. International Journal of Food Sciences and Nutrition 31(1): 33-40.

41. Subedi M (2002) Explanatory model of Food, Health and Illness Ideology in Newar Town of Kirtipur. Academiaedu.

42. Tamang A, Broom A (2010) The practice and meanings of spiritual healing in Nepal. South Asian History and Culture 1(2): 328-340.

43. Shankar R, Paudel R, Giri R (2006) Healing traditions in Nepal. Nepal India.

44. Sibeko L, Dhansay M, Charlton K, Johns T, Gray Donald K (2005) Beliefs, Attitudes, and Practices of Breastfeeding Mothers from a Peri urban Community in South Africa. Journal of Human Lactation 21(1): 31-38.

45. Fieldhouse P (1995) Customs and Culture ( $2^{\text {nd }}$ edn.).

46. Sharma S, Van Teijlingen E, Hundley V, Angell C, Simkhada P (2016) Dirty and 40 days in the wilderness: Eliciting childbirth and postnatal cultural practices and beliefs in Nepal. BMC Pregnancy and Childbirth 16(1): 147.

47. Frazer J, The golden bough A (1963) study in magic and religion.

48. Lindeman M, Keskivaara P, Roschier M (2000) Assessment of Magical Beliefs about Food and Health. Journal of Health Psychology 5(2): 195209.
49. Rozin P, Markwith M, McCauley C (1994) Sensitivity to indirect contacts with other persons: AIDS aversion as a composite of aversion to strangers, infection, moral taint, and misfortune. Journal of Abnormal Psychology 103(3): 495-504.

50. Toyama N (2000) Young children's awareness of socially mediated rejection of food. Cognitive Development 15(4): 523-541.

51. Ellahi B (2014) Dietary intake patterns of south Asian men attending mosques in Burnley UK

52. Acharya J, Teijlingen E, Murphy J, Hind M (2015) A Comparative Study on Nutritional Problems in Preschool Aged Children of Nepal. European Journal of Nutrition \& Food Safety 5(5): 389-389.

53. Pokharel R, Houston R, Harvey P (2013) Nepal Nutrition Assessment and Gap Analysis Final Report Nutrition Assessment Team, Kathmandu, Nepal: Child Health Division, MOHP.

54. Braun V, Clarke V (2006) Using thematic analysis in psychology. Qualitative Research in Psychology. 3(2): 77-101.

55. Creswell J (2014) Research Design ( $1^{\text {st }}$ edn.). Los Angeles, USA.

56. Bowling A (2014) Research methods in health: investigating health and health services ( $\left.4^{\text {th }} \mathrm{edn}\right)$. - ePrints Soton.

57. Samik-Ibrahim R (2008) Grounded Theory Methodology as the Research Strategy for a Developing Country. Nbn-resolvingde.

58. Creswell J, Plano Clark V (2011) Designing and Conducting Mixed Methods Research p. 488.

59. Shakya M (2006) Traditional Food and Health Beliefs. Education and development.

60. Subedi M (2010) Caste System: Theories and Practices in Nepal. Himalayan Journal of Sociology and Anthropology 4(0): 134-159.

61. Biza Zepro N (2015) Food Taboos and Misconceptions Among Pregnant Women of Shashemene District, Ethiopia, 2012. Science Journal of Public Health 3(3): 410.

62. Meyer-Rochow V (2009) Food taboos: their origins and purposes. Journal of Ethnobiology and Ethnomedicine 5(1): 18

63. Nagpal N (2003) Health matters: coping indigenously. Imsearlimahidolacth.

64. (2007) NPAN. National Plan for Action on Nutrition.

65. Aryal M. Stunting (2018) The Cruel Curse of Malnutrition in Nepal | Inter Press Service. Ipsnewsnet.

66. Azugbene E (2017) Maternal Health Literacy and Maternal and Child Health Outcomes: A Review of the Literature. Annals of Global Health 83(1): 94

67. (1995) Customs and Culture. 2nd ed.

68. Karkee R, Lee A, Binns C (2013) Why Women do not Utilize Maternity Services in Nepal: A Literature review.

69. Van Teijlingen E, Hundley V (2005) Pilot studies in family planning and reproductive health care. Journal of Family Planning and Reproductive Health Care 31(3): 219-221.

70. Van Teijlingen ER, Rennie AM, Hundley V, Graham W (2001) The importance of conducting and reporting pilot studies: The example of the Scottish births survey. Journal of Advanced Nursing 34(3): 289-295.

71. Pool R (1987) Hot and cold as an explanatory model: The example of Bharuch district in Gujarat, India. Social Science \& Medicine 25(4): 389399.

72. Forster D, McLachlan H, Lumley J (2006) Factors associated with breastfeeding at six months postpartum in a group of Australian women.

73. Hill S 0(1990) More Than Rice and Peas: Guidelines to improve food provision for Black and Ethnic Minorities in Britian, The Food Commission, London. Journal of Current Research 2: 103-108. 
This work is licensed under Creative Commons Attribution 4.0 License

To Submit Your Article Click Here:

Submit Article

DOI: 10.32474/LOJMS.2018.01.000119

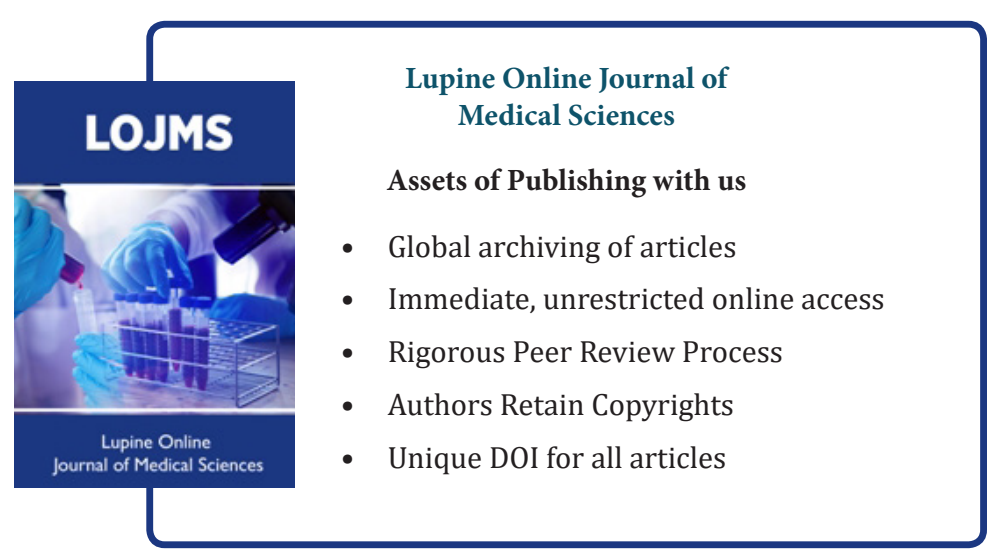

